\title{
A spectroscopic and photometric study of short-timescale variability in NGC $5548^{\star, \star \star}$
}

\author{
M. Dietrich ${ }^{1,2}$, C. F. Bender ${ }^{3}$, D. J. Bergmann ${ }^{3}$, T. E. Bills ${ }^{3}$, N. G. Bochkarev ${ }^{4}$, A. Burenkov ${ }^{5}$,
} C. M. Gaskell ${ }^{3}$, D. D. Gutzmer ${ }^{3}$, R. Grove ${ }^{3}$, M. E. Hiller ${ }^{3}$, J. P. Huchra ${ }^{6}$, E. S. Klimek ${ }^{3}$, C. Lund ${ }^{3}$, N. Merkulova ${ }^{7,8}$, S. Pebley ${ }^{3}$, M. A. Poulsen ${ }^{3}$, V. I. Pronik ${ }^{7,8}$, S. G. Sergeev ${ }^{7,8}$, E. A. Sergeeva ${ }^{7,8}$, A. I. Shapovalova ${ }^{4}$, V. V. Vlasyuk ${ }^{5}$, and B. Wilkes ${ }^{6}$

1 Department of Astronomy, University of Florida, 211 Bryant Space Science Center, Gainesville, FL 32611-2055, USA

2 Landessternwarte Heidelberg, Königstuhl, 69117 Heidelberg, Germany

3 University of Nebraska, Lincoln, Department of Physics and Astronomy, Lincoln, NE 68588-0111, USA

4 Sternberg Astronomical Institute, Universitetskij Prospect, 13, 119899 Moscow, Russia

5 Special Astrophysical Observatory, Russian Academy of Science, Nyzknij Arkhyz, Karachaj-Cherkess Republic, 369167, Russia

6 Harvard-Smithsonian Center for Astrophysics, 60 Garden Street, Cambridge, MA 02138, USA

7 Crimean Astrophysical Observatory, p/o Nauchny, 98409 Crimea, Ukraine

8 Isaac Newton Institute of Chile, Crimean Branch, Chile

Received 17 November 2000 / Accepted 6 February 2001

\begin{abstract}
Results of a ground-based optical monitoring campaign on NGC 5548 in June 1998 are presented. The broad-band fluxes $(U, B, V)$, and the spectrophotometric optical continuum flux $F_{\lambda}(5100 \AA)$ monotonically decreased in flux while the broad-band $R$ and $I$ fluxes and the integrated emission-line fluxes of $\mathrm{H} \alpha$ and $\mathrm{H} \beta$ remained constant to within $5 \%$. On June 22 , a short continuum flare was detected in the broad band fluxes. It had an amplitude of about $\sim 18 \%$ and it lasted only $\approx 90 \mathrm{~min}$. The broad band fluxes and the optical continuum $F_{\lambda}(5100 \AA)$ appear to vary simultaneously with the EUV variations. No reliable delay was detected for the broad optical emission lines in response to the EUVE variations. Narrow $\mathrm{H} \beta$ emission features predicted as a signature of an accretion disk were not detected during this campaign. However, there is marginal evidence for a faint feature at $\lambda \simeq 4962 \AA$ with $F W H M \simeq 6 \AA$ redshifted by $\Delta v \simeq 1100 \mathrm{~km} \mathrm{~s}^{-1}$ with respect to $\mathrm{H} \beta_{\text {narrow }}$.
\end{abstract}

Key words. galaxies: active - galaxies: Seyfert galaxies - galaxies: individual (NGC 5548)

\section{Introduction}

The ultraviolet and optical continuum and the broad emission line flux of Seyfert 1 galaxies are known to be variable on timescales of a few days until years. Variations on timescales of hours and even less have been observed in X-rays (e.g. Barr \& Mushotzky 1986; Leighly et al. 1996; George et al. 1998a, 1998b).

Send offprint requests to: M. Dietrich, e-mail: dietrich@astro.ufl.edu

* Based on observations taken at the German-Spanish Astronomical Centre Calar Alto, Mt. Hopkins Observatory, Crimean Observatory, SAO, University of Nebraska Observatory.

** Tables $1 \mathrm{~A}$ and $3 \mathrm{~A}$ are only available at CDS via anonymous ftp to cdsarc.u-strasbg.fr $(130.79 .128 .5)$ or via http://cdsweb.u-strasbg.fr/cgi-bin/qcct?/A+A/371/79
To study the physical processes which are responsible for the observed spectral energy distribution of an active galactic nuclei (AGN) multiwavelength monitoring campaigns have proven to be an excellent tool (cf. Peterson 1993; Netzer \& Peterson 1997 for a review; Marshall et al. 1997; Nandra et al. 1998; Edelson et al. 2000). Thus, over the last decade, several large space-based and ground-based monitoring programs have been undertaken for nearby AGN, such as NGC 5548 (Clavel et al. 1991; Peterson et al. 1991, 1992, 1994, 1999; Maoz et al. 1993; Dietrich et al. 1993; Korista et al. 1995; Chiang et al. 2000), NGC 3783 (Reichert et al. 1994; Stirpe et al. 1994; Alloin et al. 1995), NGC 4151 (Crenshaw et al. 1996; Kaspi et al. 1996; Warwick et al. 1996; Edelson et al. 1996), Fairall 9 (Rodríguez-Pascual et al. 1997; Santos-Lleó et al. 1997), 3C 390.3 (Leighly et al. 1997; Dietrich et al. 1998; O'Brien et al. 1998), and NGC 7469 (Wanders et al. 1997; Collier et al. 1998). 
A broad-line region (BLR) size of the order of less than a few light weeks is indicated for Seyfert 1 galaxies by the correlated variations of the broad emission-line flux and of the optical/ultraviolet continuum. It is generally assumed that the central supermassive black hole is surrounded by an accretion disk. Such an accretion disk is the probable origin of a significant fraction of the broad emission-line flux (e.g. Laor \& Netzer 1989; Dumont \& Collin-Souffrin 1990; Halpern 1990; Zheng et al. 1991; Hubeny et al. 2000). Recently, Kaspi et al. (2000) published results of a long term monitoring campaign of quasars. Including the results of Seyfert 1 galaxies (Wandel et al. 1999) they suggested a relation between the BLR size and the optical luminosity given by $r \sim L^{0.7}$.

The Seyfert 1 galaxy NGC 5548 has been continuously monitored in the optical since late 1988 by the international AGN watch consortium (Peterson et al. 1999; cf. Alloin 1994 for an AGN watch overview). In June 1998 this prominent Seyfert 1 galaxy was targeted for a coordinated intense monitoring campaign using EUVE, ASCA, and RXTE to study the high energy continuum emission and its temporal characteristics (Chiang et al. 2000). Chiang et al.'s observations indicate that the variations at $\sim 0.2 \mathrm{keV}$ (EUV) appear to lead similar variations at energies larger than $\sim 1 \mathrm{keV}$ by $3-8 \mathrm{hrs}$. This was unexpected as it was generally assumed that correlated variations of the EUV, UV, and optical emission would all be due to reprocessed higher energy radiation.

Since this campaign provided the rare opportunity to access the high energy continuum variations with especially high temporal sampling, we organized a simultaneous ground-based campaign for the optical wavelength domain.

Assuming part of the BLR flux is emitted from the accretion disk, variations on timescales of one day or even less should be detected (Stella 1990). For this model of a relativistic Keplerian disk it was shown that weak narrow features should drift across the emission line profile. These weak structures are expected to start at high velocities i.e. in the outer profile wings, and move towards the line center to be due to the longer time delay at larger radii of the disk in response to the variable continuum emission. The timescale of the shift depends on the mass of the central black hole and it is for NGC 5548 of the order of less than several days (cf. Stella 1990). The detection of such structures would be a strong indication for the presence of an accretion disk in the innermost region of an AGN.

Generally, current monitoring campaigns have not provided the necessary temporal and spectral resolution for detecting such substructures in the broad emission line profiles. However short term monitoring campaigns had been undertaken for NGC 4151 (Xanthopoulos \& DeRobertis 1991; Crenshaw et al. 1996; Kaspi et al. 1996; Warwick et al. 1996; Edelson et al. 1996) and for a small sample of AGN (e.g. NGC 5548, NGC 4151, 3C 390.3, Arp102B, Mkn 6; Eracleous \& Halpern 1993). As yet no significant short timescale broad emission line flux variation or profile variability has been detected.
In this paper, we present the results of the optical photometric and spectroscopic observations that were obtained in June 1998 simultaneous with the high energy campaign (Chiang et al. 2000). In Sect. 2 we describe the optical observations and outline intercalibration procedures by which a homogeneous set of photometric and spectroscopic measurements can be achieved. In Sect. 3 we present measurements of the broad-band flux as well as of the broad Balmer emission-line flux. We compare our results with the results of the simultaneous campaign of the EUV wavelength range (Chiang et al. 2000). We also discuss the shape of the $\mathrm{H} \alpha$ and $\mathrm{H} \beta$ line profiles. We summarize our results in Sect. 4.

\section{Observations and data analysis}

Spectroscopic and broad-band photometric measurements of NGC 5548 were obtained by several groups in June 1998. Table 1 gives a brief overview of the individual participants who recorded the data we report here. Each group (Col. 1) was assigned an identification code given in Col. (2) which is used throughout this paper. This code was based on that used for the ongoing NGC 5548 monitoring campaign (Peterson et al. 1999). Column (3) gives the aperture of the telescope used. Columns (4)-(8) list the sizes of the focal-plane apertures used in various observations; fixed instrument apertures were used. In Col. (9), the spectrograph slit width (in the dispersion direction) and extraction width (cross-dispersion dimension) of the spectra are listed.

A complete log of the photometric and spectroscopic observations is given in Tables 1A (available in electronic form at the CDS) and $2 \mathrm{~A}$.

\subsection{Optical photometry}

Photometric observations of NGC 5548 were obtained by Gaskell et al. (sample Q) and Merkulova (sample D). The brightness of NGC 5548 was determined with respect to stars in the field of NGC 5548 using the photometric sequence (stars 1 and 2) defined by Penston et al. (1971), and star C1 and C defined by Lyuty (1972). The bright star $\approx 1^{\prime}$ east to the galaxy located at $\mathrm{PA}=240^{\circ}$ at a distance of $\approx 9^{\prime}$ with respect to NGC 5548 also was used. This star is referred in the HST guidestar catalogue as gsc $0201001062\left(\mathrm{RA}=17^{\mathrm{h}} 17^{\mathrm{m}} 29.66\right.$, Dec $=+25^{\circ} 03^{\prime} 10^{\prime \prime} \cdot 5$ (2000.0)). Star C1 in Lyuty is identical to star 4 in Penston et al. The $U, B, V$ magnitudes of $\mathrm{C} 1$ and 4 are identical within the errors and differ by only $\pm 0.02 \mathrm{mag}(B, V)$ and $\pm 0.17 \mathrm{mag}(U)$ (cf. Table 2). Star $\mathrm{C}$ is the bright star close to star 3 in Penston et al. located approximately 1'3 southeast of it.

The photometric observations of NGC 5548 of sample D obtained with the $1.25 \mathrm{~m}$ telescope of the Crimean Astrophysical Observatory were recorded using a five channel version of the Double Image Chopping Photometer - Polarimeter (Piirola 1973). The observations were made simultaneously in five colors using 
Table 1. Overview of observations; the aperture size is given in units of arcsec

\begin{tabular}{lccccccccc}
\hline Source & Code & \multicolumn{1}{c}{ Tel. } & \multicolumn{3}{c}{ Photometry Aperture } & Spectroscopy \\
& & {$[\mathrm{m}]$} & $U$ & $B$ & $V$ & $R$ & $I$ & Aperture \\
\hline Mt. Hopkins Observatory & $\mathrm{C}$ & 1.5 & - & - & - & - & - & $3 \times 4.6$ \\
Crimean Astrophysical Obs. & $\mathrm{D}$ & 1.25 & 15 & 15 & 15 & 15 & 15 & - \\
Calar Alto Observatory & $\mathrm{G}$ & 2.2 & - & - & - & - & - & $2.06 \times 2.61$ \\
Special Astrophysical Obs. & L1 & 1.0 & - & - & - & - & - & $8 \times 6.6,8 \times 19.8$ \\
Special Astrophysical Obs. & L2 & 6.0 & - & - & - & - & - & $2 \times 6$ \\
Univ. of Nebraska $0.4 \mathrm{~m}$ & $\mathrm{Q}$ & 0.4 & - & - & 8 & - & - & - \\
Shajn reflector, Crimean Obs. & W & 2.6 & - & - & - & - & - & $3 \times 11$ \\
\hline
\end{tabular}

Table 2. Adopted magnitudes for standard stars

\begin{tabular}{cccccc}
\hline band & $1^{a}$ & $2^{a}$ & $4, \mathrm{C}^{b}$ & $\mathrm{C}^{b}$ & 0 \\
\hline$U$ & 14.52 & 16.24 & 13.23 & 11.17 & - \\
$B$ & 14.45 & 16.07 & 12.78 & 10.96 & - \\
$V$ & 13.80 & 15.38 & 11.90 & 10.47 & 11.29 \\
$R$ & - & - & 11.17 & 9.96 & - \\
$I$ & - & - & 10.64 & 9.57 & - \\
\hline
\end{tabular}

${ }^{a}$ From Penston et al. (1971), estimated uncertainty 0.02 mag.

${ }^{b}$ From Lyuty (1972), estimated uncertainty $0.01 \mathrm{mag}$. $(U)$ of C1 0.02 mag.

dichroic filters to split the light into five spectral bands. The resulting passbands are close to the standard Johnson $U B V R I$ photometric system with effective wavelengths at $3600 \AA, 4400 \AA, 5300 \AA, 6900 \AA$, and $8300 \AA$, respectively. A diaphragm with two equal apertures $\left(15^{\prime \prime}\right.$ in diameter) was used in the focal plane of the $1.25 \mathrm{~m}$ telescope. The distance between the aperture centers was $26^{\prime \prime}$. A rotating chopper alternately closes one of the apertures, leaving the other free, thus the photocathode is illuminated alternately by the galaxy (or the star) and the sky apertures. Because the centers of the apertures are closely spaced, background observations were also obtained at a distance of about $7^{\prime}$ from the galaxy nucleus to correct the observational background for a contribution of the outer regions of the galaxy. The telescope is fully automated and an autoguider was used. Observations of the nucleus of NGC 5548 were made on nights with good atmospheric conditions when the estimated seeing was in the range of $1^{\prime \prime}$ to $3^{\prime \prime}$. The positional accuracy by the autoguider during observations is better than $20 \%$ of the estimated seeing, i.e. better than $0 . ! 6$ in the worst case. The galaxy nucleus was also positioned in the aperture using the autoguider, with similar positioning errors. The conventional technique of differential measurements was applied. Two comparison stars (labelled as C1 and C) were taken from the list of Lyuty (1972). Observations were performed against a comparison star C1; and the second star, C, was used to check the results of the first comparison. In addition to stars $\mathrm{C} 1$ and C, secondary $U B V R I$ standards by Neckel \& Chini (1980) were used for an absolute calibration. The measurements were carried out by observing in the following sequence, $\mathrm{C}-\mathrm{C}_{1}-$ sky-AGN-sky- $\mathrm{C}_{1}-\mathrm{C}-$-sky. The time resolution was about 3.5 min. During a single observation, 8 integrations of $10 \mathrm{~s}$ each were made. Photon statistics (corrected for sky background) were applied to calculate photometric errors, which generally were the same as the rms errors obtained by averaging the 8 integrations. Generally, the atmospheric seeing during the observations obtained with the $1.25 \mathrm{~m}$ CAO telescope averaged $2^{\prime \prime}$. It was better for two nights (June $27 / 28$ and June 30/July 1 ) and worse for the night June $22 / 23\left(2-3^{\prime \prime}\right)$.

The $V$-band observations of Gaskell et al. (sample Q) were obtained with the University of Nebraska $0.4 \mathrm{~m}$ telescope in Lincoln, Nebraska. The frames were recorded with a ST-7 CCD camera. The flux of NGC 5548 was determined for an $8^{\prime \prime}$ diameter aperture. The sky was measured in an annulus of radius 15 to $20^{\prime \prime}$. The $V$-band magnitude was derived relative to the comparison stars 1 and gsc0201001062 in the field of NGC 5548 (cf. Penston et al. 1971). The exposure time of an individual frame was $3 \mathrm{~min}$. Generally, to increase the signal-to-noise ratio of the flux measurements, the $V$-band magnitudes of three subsequently recorded frames were averaged. The seeing during the observations was typically $4^{\prime \prime}$. There was no correlation detected between the derived magnitude of NGC 5548 and the seeing.

To combine the broad band flux measurements of sample D and Q, the apparent magnitudes were transformed into fluxes. The conversion was performed using the following equations (Allen 1973; Wamsteker 1981):

$\log F_{\lambda}(3600 \AA)=-0.4 m_{U}-8.361$,

$\log F_{\lambda}(4400 \AA)=-0.4 m_{B}-8.180$,

$\log F_{\lambda}(5500 \AA)=-0.4 m_{V}-8.439$,

$\log F_{\lambda}(7000 \AA)=-0.4 m_{R}-8.759$,

$\log F_{\lambda}(9000 \AA)=-0.4 m_{I}-9.080$.

The broad band $V$-fluxes of sample Q were scaled to the $V$-band fluxes observed for sample $\mathrm{D}$. The additive scaling factor was determind by comparison of observations which were separated by less than 2 hours. We derived a scaling factor of $(2.944 \pm 0.524) 10^{-15} \mathrm{erg} \mathrm{s}^{-1} \mathrm{~cm}^{-2} \AA^{-1}$ based on 5 epochs which corresponds to $\Delta$ mag $=0.20 \pm 0.02$. The difference is caused by the significantly different sizes 

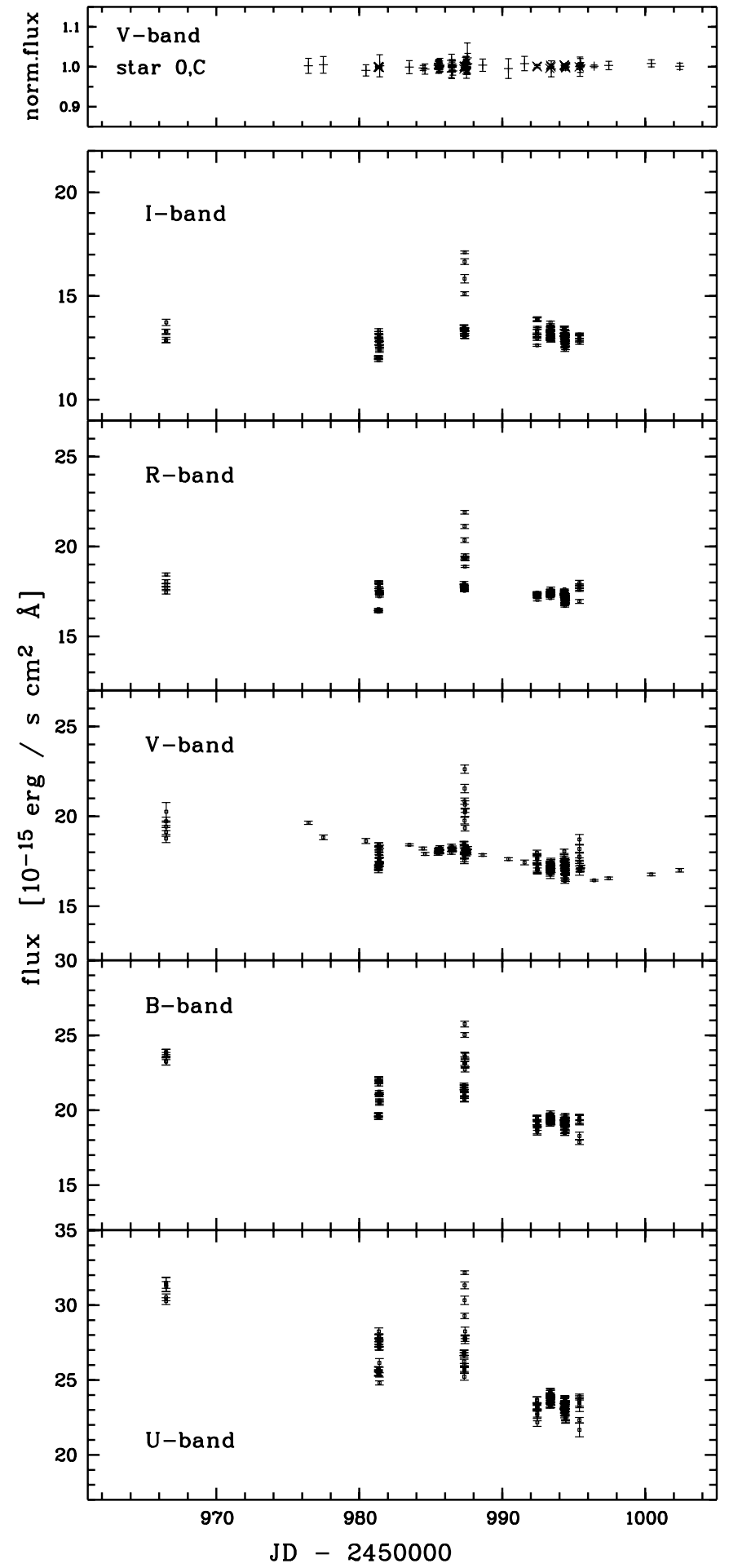

Fig. 1. Optical broad-band light curves for NGC 5548. Fluxes in broad-band $U, B, V, R$, and $I$ are in units of $10^{-15} \mathrm{erg} \mathrm{s}^{-1} \mathrm{~cm}^{-2} \AA^{-1}$. In the top panel the normalized $V$ band flux of star $0(+)$ and $\mathrm{C}(\mathrm{x})$ is shown which was contant within $\sim 0.57 \%$

of the apertures used to measure the flux of NGC 5548 (Table 1). The resulting $U, B, V, R, I$ broad-band light curves are given in Table 1A (available in electronic form at the CDS) in units of $10^{-15} \mathrm{erg} \mathrm{s}^{-1} \mathrm{~cm}^{-2} \AA^{-1}$ and are displayed in Fig. 1.

\subsection{Optical spectroscopy}

The flux calibration of AGN spectra can be accomplished in several ways. In variability studies, it has become common practice to normalize the flux scale to the fluxes of strong forbidden narrow emission lines which are assumed to be constant over timescales of at least several decades (e.g. Peterson 1993). This assumption is justified by the large spatial extent and low gas density of the narrow-line region (NLR), but is only valid if the aperture used is larger than the NLR. Light travel-time effects and the long recombination timescale $\left(\tau_{\text {rec }} \approx 100\right.$ years for $n_{\mathrm{e}} \approx 10^{3} \mathrm{~cm}^{-3}$ ) damp out short timescale variability.

As a major sources of uncertainty in inhomogenous samples of variability data is the use of different instrumental settings, it is important to take aperture effects into account (Peterson \& Collins 1983). The seeingdependent uncertainties which are introduced by the aperture geometry can be minimized by using large apertures. It has been shown that apertures of $5^{\prime \prime} \times 7^{\prime \prime} .5$ can reduce seeing-dependent photometric errors to no more than a few percent in the case of nearby AGNs (Peterson et al. 1995).

The spectra of NGC 5548 obtained at the $1 \mathrm{~m}$ telescope of SAO RAS (sample L1 Table 1) were extracted for a slit aperture of $8^{\prime \prime} .0 \times 6$. $^{\prime \prime} 6$ and $8^{\prime \prime} .0 \times 199^{\prime \prime} 8$, respectively. For these large apertures seeing losses can be neglected. To measure the $F([\mathrm{O}$ III $] 5007 \AA)$ a linear pseudocontinuum was fitted beneath the [O III] emission line and the flux was measured for the wavelength range $\lambda \lambda 5062-5108 \AA$ A. For the extraction windows used for sample L1 we determined a flux of $F([$ O III $] 5007 \AA)=$ $(5.07 \pm 0.05) \quad 10^{-13} \mathrm{erg} \mathrm{s}^{-1} \mathrm{~cm}^{-2}\left(8^{\prime \prime} .0 \times 66^{\prime \prime} 6\right)$ and $F([\mathrm{O} \mathrm{III}] 5007 \AA)=(5.91 \pm 0.05) \quad 10^{-13} \mathrm{erg} \mathrm{s}^{-1} \mathrm{~cm}^{-2}$ $\left(8{ }^{\prime \prime} 0 \times 19^{\prime \prime} 8\right)$, respectively. These values are in good agreement with $F([\mathrm{O}$ III $] 5007 \AA)$ measured for NGC 5548 during the last 10 years (cf. Peterson et al. 1999). For large apertures of comparable size (e.g. 8.". $8 \times 12^{\prime \prime} .8$ ) $F([\mathrm{O}$ III $] 5007 \AA)=5.1$ to $5.910^{-13} \mathrm{erg} \mathrm{s}^{-1} \mathrm{~cm}^{-2}$ is mentioned (Peterson et al. 1992). To be constistent with the $\mathrm{H} \beta$ line flux measurements of NGC 5548 obtained since 1988 (Peterson et al. 1999) we adopted $F([\mathrm{O} \mathrm{III}] 5007 \AA)=(5.58 \pm 0.27) 10^{-13} \mathrm{erg} \mathrm{s}^{-1} \mathrm{~cm}^{-2}$.

\subsection{Intercalibration of the spectra}

Since the spectra of the various samples were taken with different instruments in different configurations, it was necessary to intercalibrate them to a common flux level. As we have shown above, the [O III] 5007 line flux was constant to better than $1 \%$ for large extraction windows. We can safely use the narrow emission lines such as the flux of [O III] 5007 as standards. In order to avoid any wavelength-dependent calibration errors, each spectrum was scaled in flux locally over a limited wavelength range prior to measurement. The $\mathrm{H} \beta$ spectral region was scaled with respect to the [O III] 4959, 5007 line fluxes. The $\mathrm{H} \alpha$ region was scaled with respect to the flux of the [O I] 6300, 
[N II] 6548, 6583, and [S II] 6716, 6731 emission lines. The spectra were intercalibrated using the method described by van Groningen \& Wanders (1992). This procedure corrects for different flux scales, small wavelength shifts, and different spectral resolutions by minimizing the narrowline residuals in difference spectra formed by subtracting a "reference spectrum" from each of the observed spectra. The rescaled spectra were used to derive integrated emission-line fluxes.

\section{Results}

\subsection{Light curves}

The broad band flux variations in $U, B, V, R, I$ are displayed in Fig. 1. In addition to the broad band flux variations, the light curve of the measured flux of the comparison stars $\mathrm{C}$ and 0 (cf. Table 2) are shown. The normalized flux remained constant within $\sim 0.57 \%$. The broad band flux of NGC 5548 decreases throughout the campaign in June 1998. This trend is clearly visible in the $U$ and $B$-band, and the ratio of the flux levels observed in early and late June becomes smaller for increasing wavelength. In the $I$-band the flux decay is negligible. The merged $V$-band light curve of sample $\mathrm{D}$ and $\mathrm{Q}$ shows different internal scatter of the measurements recorded during individual nights (Figs. 1, 2). This difference might be caused by different seeing conditions of the observations. Merkulova (sample D) measured the brightness of NGC 5548 with an $15^{\prime \prime}$ aperture under a typical seeing of $1-3^{\prime \prime}$. The seeing amounts to $\approx 4^{\prime \prime}$ for the measurements Gaskell et al. (sample Q) observed for an $8^{\prime \prime}$ aperture. Modelling the host galaxy of NGC 5548 Romanishin et al. (1995) showed that the light loss of the AGN is of the order of $10 \%$ for sample Q and only slightly larger for the host galaxy. However, the different scatter may be also intrinsic. The temporal resolution of the samples amounts to $\leq 4 \mathrm{~min}(\mathrm{D})$ and nearly $16 \mathrm{~min}(\mathrm{Q})$, respectively. The scatter of the brightness during individual nights is $\approx 5 \%$ for sample D. This amplitude is of the order which is expected for microvariability. For several Seyfert 1 galaxies (NGC 3516, NGC 4151, NGC 5548, NGC 7469) detection of microvariability was claimed on timescales of less than 10 min (cf. Lyuty et al. 1989; Dultzin-Hacyan et al. 1992; Jang \& Miller 1997; Noble et al. 1997; Carini et al. 1998; Welsh et al. 1998; Ghosh et al. 2000; Merkulova 2000) and amplitudes of the order of $15 \%$ to $20 \%$. However, it seems that onset and disappearance of microvariability follow a random process since several attempts to detect or to confirm rapid optical variations of Seyfert 1 galaxies failed (cf. Dultzin-Hacyan et al. 1993; Petrucci et al. 1999). Hence, it might be that rapid variations can not be detected on timescales larger than $15 \mathrm{~min}$.

The most obvious structure in the broad band light curves is a flare like event. This variation was detected on JD $=2450987$ (June 22). The observations recorded during this night are shown in more detail in Fig. 2. In all 5 broad band measurements a short intense increase of
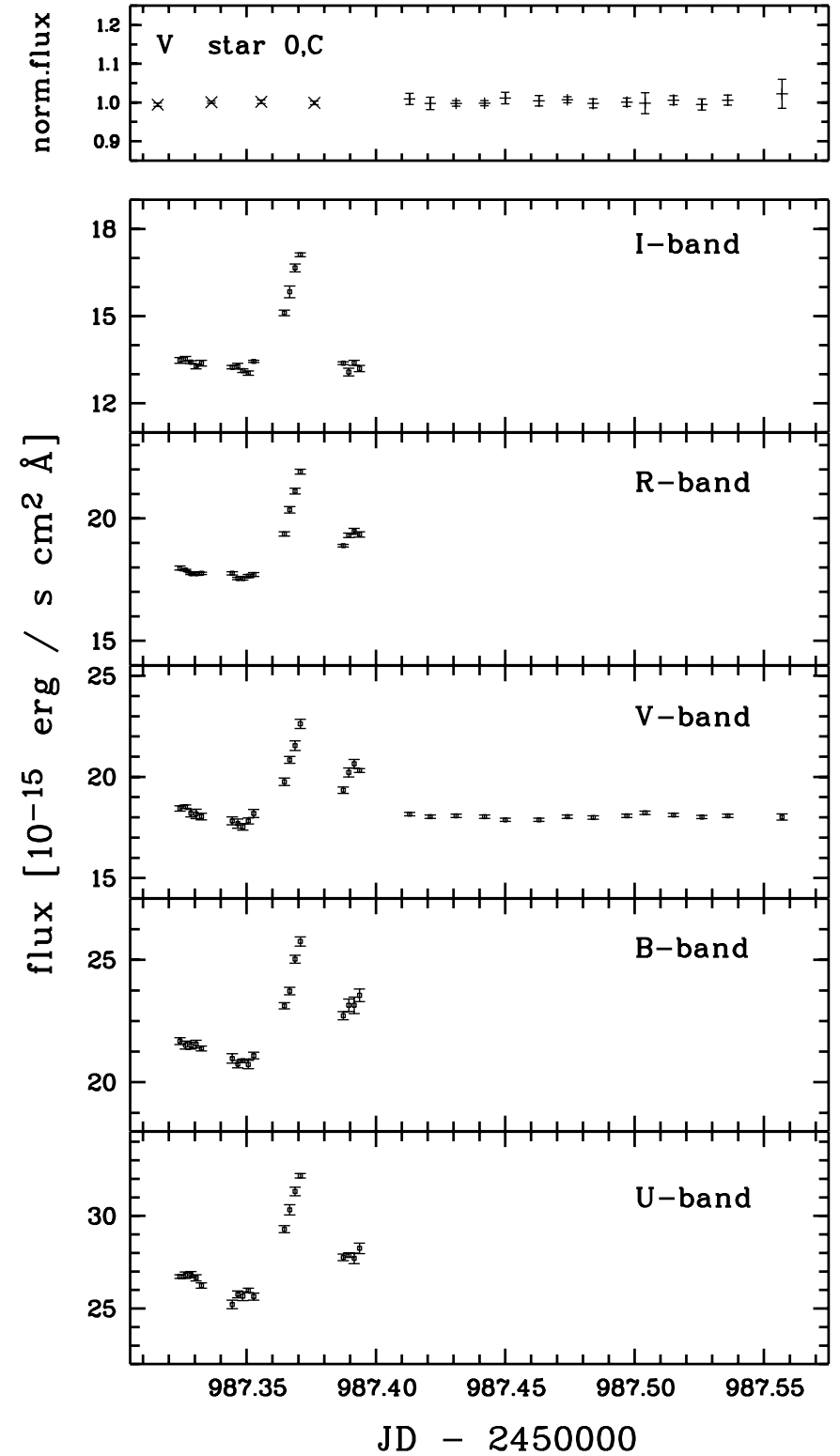

Fig. 2. The strong variation in the broad band fluxes as measured on June 22 (JD = 2450987). In all 5 broad band fluxes a rapid increase and subsequent decay was detected within $\approx 90$ min. In the top panel the normalized $V$-band flux of star $0(+)$ and $\mathrm{C}(\mathrm{x})$ is shown

the flux level occured. The broad band flux increased by $20 \pm 2 \%$ within $\approx 30$ min and decreased within $\approx 60 \mathrm{~min}$ to the flux level of the beginning of this event. The duration of this flare lasted only $\approx 90 \mathrm{~min}$. This remarkable flux increase can not be due to temporal variations of a standard star which was used for relative flux calibration. The flux calibration is based on two stars which were in cross checked with secondary standard stars taken from Neckel \& Chini (1980) in addition. As can be seen in Figs. 1 and 2 the used comparison stars were constant within less than $0.57 \%$. The standard deviations of the primary star used for calibration during this night amount to $0.018,0.014$, 0.009, 0.023, $0.001 \mathrm{mag}$ in $U, B, V, R, I$. 


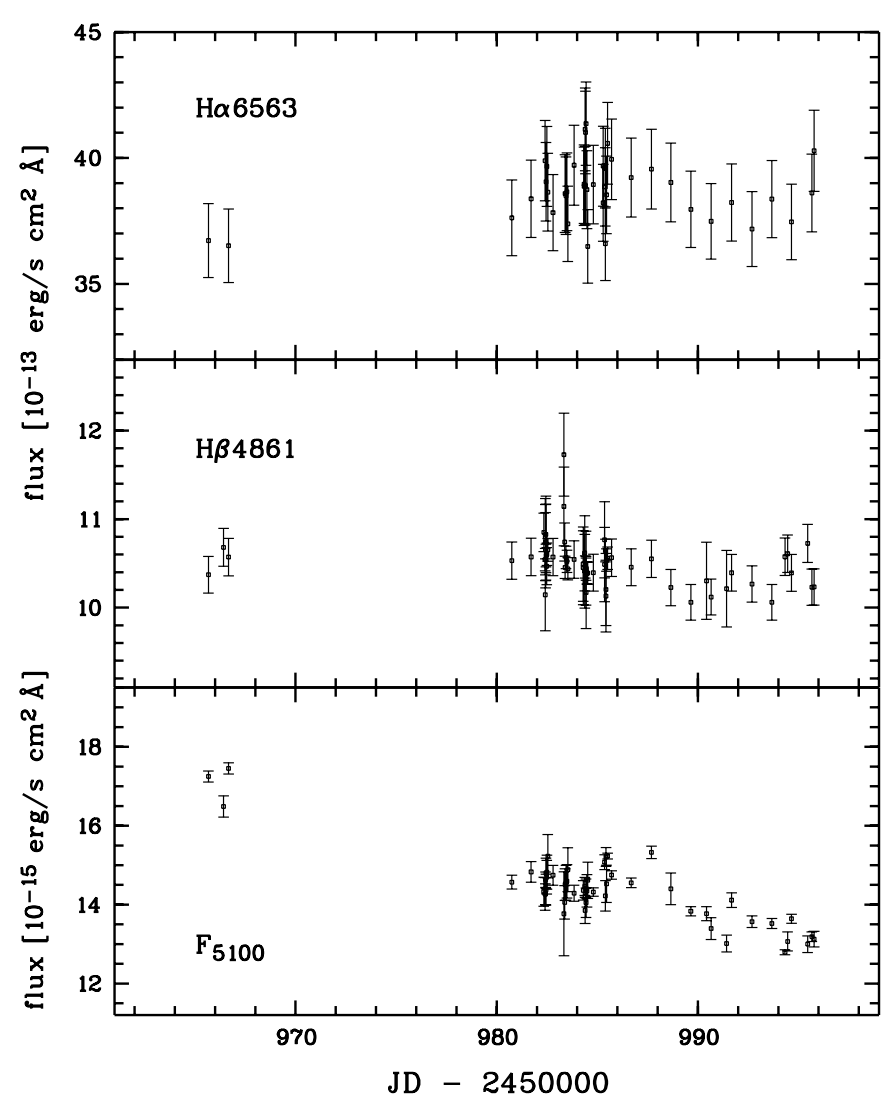

Fig. 3. Light curves for the emission lines $\mathrm{H} \alpha, \mathrm{H} \beta$, and the optical continuum flux $F_{\lambda}(5100 \AA)$. The vertical scale is in units of $10^{-13} \mathrm{erg} \mathrm{s} \mathrm{cm}^{-1} \AA^{-1}$ for the lines and $10^{-15} \mathrm{erg} \mathrm{s}^{-1} \mathrm{~cm}^{-2} \AA^{-1}$ for the continuum

Broad emission-line fluxes were integrated over a wavelength range of $\lambda \lambda 4850-5020 \AA$ for $\mathrm{H} \beta$ and $\lambda \lambda 6500-6800 \AA$ for $\mathrm{H} \alpha$. A local linear continuum fit was interpolated under each emission line. In the case of the $\mathrm{H} \beta$ region, the continuum was defined by the flux measured in two windows $(10 \AA$ width) at $4845 \AA$ and $5170 \AA$ in the observed frame $(\mathrm{H} \beta)$ and at $6350 \AA$ and $6965 \AA(\mathrm{H} \alpha)$. No attempt was made to correct any of the measured emission-line fluxes for their respective narrow-line contributions. The optical continuum flux $F_{\lambda}(5100 \AA)$ was determined as the average value in the range 5185-5195 $\AA$ (cf. Table 4).

The measured $\mathrm{H} \beta$ and $\mathrm{H} \alpha$ emission line fluxes were adjusted to take into account the different slit sizes (Table 1). The measurements of both $\mathrm{H} \beta$ and $\mathrm{H} \alpha$ were normalized by a similar factor

$F_{\mathrm{H} \beta}=\varphi F_{[\mathrm{O} \mathrm{III}]}\left[\frac{F_{\mathrm{H} \beta}}{F_{[\mathrm{O} \mathrm{III}]}}\right]_{\mathrm{obs}}$.

The continuum fluxes $F_{\lambda}(5100 \AA)$ were then adjusted for different amounts of host-galaxy contamination (see Peterson et al. 1995 for a detailed discussion) by

$F_{\lambda}(5100 \AA)=\varphi F_{[\mathrm{O} \mathrm{III}]}\left[\frac{F_{\lambda}(5100 \AA)}{F_{[\mathrm{O} \mathrm{III}]}}\right]_{\mathrm{obs}}+G$,

where $F_{[\mathrm{OIII}]}$ is the adopted absolute flux of $F$ ([O III] $5007 \AA)$, the quantity in brackets is the observed
Table 3. Scaling factors for photometric and spectroscopic subsets

\begin{tabular}{|c|c|c|c|}
\hline sample & $\begin{array}{l}\text { add. constant } \\
\qquad-\mathrm{mag}^{a}\end{array}$ & $\begin{array}{l}\text { point source } \\
\text { correction } \varphi^{b}\end{array}$ & $\begin{array}{c}\text { extended source } \\
\text { correction } \mathrm{G}^{b}\end{array}$ \\
\hline $\mathrm{C}$ & - & $0.972 \pm 0.022$ & $2.02 \pm 0.33$ \\
\hline $\mathrm{D}$ & 1.000 & - & - \\
\hline $\mathrm{G}$ & - & $0.865 \pm 0.022$ & $3.36 \pm 0.54$ \\
\hline L1 & - & 1.00 & 0.00 \\
\hline L2 & - & $0.945 \pm 0.025$ & $2.60 \pm 0.53$ \\
\hline Q & $2.944 \pm 0.524$ & - & - \\
\hline W & 一 & $0.950 \pm 0.028$ & $1.52 \pm 0.32$ \\
\hline
\end{tabular}

${ }^{a}$ Relative to sample D.

${ }^{b}$ Relative to sample L1.

Table 4. Integration limits

\begin{tabular}{lc}
\hline feature & $\begin{array}{c}\text { wavelength range } \\
{[\AA]}\end{array}$ \\
\hline $\mathrm{H} \alpha \lambda 6563$ & $6500-6800$ \\
$\mathrm{H} \beta \lambda 4861$ & $4850-5020$ \\
$F_{\lambda}(5100 \AA)$ & $5185-5195$ \\
\hline
\end{tabular}

continuum to $F([\mathrm{O}$ III $] 5007 \AA$ ) flux ratio measured from the spectrum, and $G$ is an aperture-dependent correction for the host-galaxy flux. The sample L1 which uses a relatively large aperture $\left(8 . \prime 0 \times 6{ }^{\prime \prime} 6\right)$ was adopted as the standard (i.e., $\varphi=1.0, G=0$ by definition), and other data sets were merged progressively by comparing measurements based on observations made during the same night. Note that this means that any real variability that occurs on timescales this short will be somewhat suppressed by the process that allows us to combine the different data sets. The scaling factor $\varphi$ and the additive scaling factor $G$ for the various samples are given in Table 3 .

The resulting light curves of the optical continuum $F_{\lambda}(5100 \AA)$ and of the $\mathrm{H} \beta$ and $\mathrm{H} \alpha$ emission line fluxes are displayed in Fig. 3 and presented in Table $3 \mathrm{~A}$ (available in electronic form at the CDS). The optical continuum light curve is very similar to the broad-band $V$ light curve (cf. Figs. 1 and 3). The difference in the flux levels is probably due to the larger aperture used for the broad band flux determination relative to that used for the optical continuum fluxes.

A final check of the uncertainty estimates was performed by examining the ratios of all pairs of photometric and spectroscopic observations which were separated by 0.05 days or less.

There are more than a dozen independent pairs of measurements separated by less than $0.05 \mathrm{~d}$. For $\mathrm{H} \alpha$, the uncertainty estimate is dominated by the spectra taken at Calar Alto Observatory, while for $\mathrm{H} \beta$ more than 20 pairs could be used. The dispersion about the mean (unity), divided by $\sqrt{2}$, provides an estimate of the typical uncertainty in a single measurement $\left(\sigma_{\text {est }}\right)$. The observational 
Table 5. Comparison of uncertainty estimates

\begin{tabular}{lcclcc}
\hline feature & $\sigma_{\text {obs }}^{a}$ & $\sigma_{\text {est }}^{b}$ & feature & $\sigma_{\text {obs }}^{a}$ & $\sigma_{\text {est }}^{b}$ \\
\hline$U$ & 0.008 & 0.021 & $I$ & 0.006 & 0.024 \\
$B$ & 0.008 & 0.020 & $F_{\lambda}(5100 \AA)$ & 0.020 & 0.016 \\
$V$ & 0.010 & 0.020 & $\mathrm{H} \beta$ & 0.022 & 0.010 \\
$R$ & 0.004 & 0.016 & $\mathrm{H} \alpha$ & 0.040 & 0.025 \\
\hline
\end{tabular}

${ }^{a}$ Observational uncertainty based on uncertainties assigned to individual points.

${ }^{b}$ Mean fractional uncertainty based on point-to-point differences between closely spaced (i.e., $\Delta t \leq 0.05 \mathrm{~d}$ ) measurements.

uncertainties $\left(\sigma_{\text {obs }}\right)$ assigned to the spectral line flux measurements were estimated from the error spectra which were calculated by the intercalibration routine, as well as from the signal-to-noise ratio within the spectral range near the individual emission lines. For example the mean fractional flux error is $\sigma_{\mathrm{obs}}=0.040$ for the $\mathrm{H} \alpha$ line, and the average fractional uncertainty from the internal statistical estimate is $\sigma_{\text {est }}=0.025$. For the $\mathrm{H} \beta$ line the values are $\sigma_{\text {obs }}=0.022$ and $\sigma_{\text {est }}=0.010$ which implies that the error estimates for both lines are probably quite good. Generally, the estimated errors $\left(\sigma_{\text {est }}\right)$ are of the same order as, but slightly smaller than, the observational uncertainties $\left(\sigma_{\text {obs }}\right)$ derived directly from multiple measurements (Table 5). The large difference of $\sigma_{\text {obs }}$ and $\sigma_{\text {est }}$ for the broad band photometric fluxes is mainly due to the measurements obtained on JD = 2450987 (June 22), which we expect is due to a real variation.

The average interval between measurements of the combined broad band light curves is about $0.24 \pm 1.53$ days for $U, B, R, I$, and for $V 0.20 \pm 0.87$ days. However, if the large gaps ( $>5$ days) are not taken into account, the sampling rate drops to $0.03 \pm 0.15$ days $(U, B, R$, $I)$ and $0.15 \pm 0.47$ days $(V)$ (Table 6$)$. The sampling of the $\mathrm{H} \alpha, \mathrm{H} \beta$, and the optical continuum flux $F_{\lambda}(5100 \AA)$ can be obviouly devided into two regimes. Taking into account all measurements the sampling is about $\Delta t(\mathrm{H} \beta)=$ $0.59 \pm 1.96$ days for $\mathrm{H} \beta$ and $F_{\lambda}(5100 \AA)$. Neglecting the large temporal gap of nearly two weeks at the beginning of the monitoring campaign (full moon), $\Delta t$ is $\Delta t(\mathrm{H} \beta)=$ $0.32 \pm 0.36$ days. For the $\mathrm{H} \alpha$ light curve the corresponding sampling rates are $\Delta t(\mathrm{H} \alpha)=0.81 \pm 2.28$ days and $\Delta t(\mathrm{H} \alpha)=0.45 \pm 0.43$ days, respectively.

The broad $\mathrm{H} \alpha$ and $\mathrm{H} \beta$ emission lines, the optical continuum flux $F_{\lambda}(5100 \AA)$ and the $U, B, V, R, I$ broad band fluxes appear to exhibit small amplitude variations on timescales of days (Figs. 1-3). The broad band light curves $U, B, V$, as well as the $F_{\lambda}(5100 \AA)$ flux show a decreasing flux from the beginning of this campaign to the end. The broad band flux at longer wavelengths $(R$ and $I)$ appeared to be nearly constant.

The $\mathrm{H} \alpha$ and $\mathrm{H} \beta$ light curves show weak evidence for small amplitude variations on timescales of only a few days. However, this is statistically not significant; within the errors the $\mathrm{H} \alpha$ and $\mathrm{H} \beta$ emission line flux can be
Table 6. Sampling characteristics

\begin{tabular}{lccc}
\hline feature & $N$ & $\begin{array}{c}\text { interval } \\
\text { [days] }\end{array}$ & $\begin{array}{c}\text { interval }^{a} \\
\text { [days] }\end{array}$ \\
\hline$U$ & 120 & $0.24 \pm 1.53$ & $0.03 \pm 0.15$ \\
$B$ & 120 & $0.24 \pm 1.53$ & $0.03 \pm 0.15$ \\
$V$ & 178 & $0.20 \pm 0.87$ & $0.15 \pm 0.47$ \\
$R$ & 120 & $0.24 \pm 1.53$ & $0.03 \pm 0.15$ \\
$I$ & 120 & $0.24 \pm 1.53$ & $0.03 \pm 0.15$ \\
$F_{\lambda}(5100 \AA)$ & 52 & $0.59 \pm 1.96$ & $0.32 \pm 0.36$ \\
$\mathrm{H} \beta$ & 52 & $0.59 \pm 1.96$ & $0.32 \pm 0.36$ \\
$\mathrm{H} \alpha$ & 38 & $0.81 \pm 2.28$ & $0.45 \pm 0.43$ \\
\hline
\end{tabular}

${ }^{a}$ Neglecting temporal gaps larger than 5 days.

regarded as nearly constant. This can be seen by deriving the variability parameter $F_{\text {var }}$.

The variability parameters $F_{\text {var }}$ and $R_{\max }$ have been calculated for the broad-band flux variations as well as for the broad emission lines and the optical continuum (cf. Clavel et al. 1991; Rodríguez-Pascual et al. 1997). The quantity $R_{\max }$ is the ratio of the maximum to the minimum flux. The quantity $F_{\mathrm{var}}$ is an estimation of the fluctuations of the intrinsic variations relative to the mean flux. Therefore, the rms of the light curves has been corrected with respect to the uncertainties introduced by the observations. Values of $R_{\max }$ and $F_{\text {var }}$ for the broad-band measurements are given in Table 7 . The value of $F_{\text {var }}$ for the optical continuum $F_{\lambda}(5100 \AA)$ agrees well with $F_{\text {var }}$ for the $V$-band. Furthermore, for the broad band variations and the optical continuum we reproduce the well known trend that the amplitude of the variation decreases with increasing wavelength (Table 7 ). The broad band fluxes and the $F_{\lambda}(5100 \AA)$ continuum flux were not corrected for the host galaxy contribution since this fraction is only of the order of less than 10\% (Romanishin et al. 1995). The values of $F_{\text {var }}$ determined for the $\mathrm{H} \alpha$ and $\mathrm{H} \beta$ emission line flux variations indicated that the Balmer line flux can be taken as constant within the errors, even if one is tempted to glimpse hints of small amplitude variations on timescales of a few days (cf. Table 7, Fig. 3).

For comparison we also calculated $F_{\text {var }}$ and $R_{\max }$ for the rapid variations detected by Chiang et al. (2000) in the extrem ultraviolet domain (Table 7). In Fig. 4 we show the normalized light curve of $F_{\lambda}(5100 \AA)$ and of the EUV variations (kindly provided by Chiang and collaborators). The $F_{\lambda}(5100 \AA)$ continuum flux show the same overall trend as the EUV variations. But in the optical continuum the rapid variations visible in the EUV are smeared out or the amplitude of the variations at $\lambda=5100 \AA$ is so small that it is hidden by the measurement uncertaintities. The disappearance of small scale variations with increasing wavelength was also observed for NGC 4151 in Dec. 1993 (cf. Edelson et al. 1996). 
Table 7. Variability statistics of the light curves; continuum flux and broad band fluxes in units of $10^{-15} \mathrm{erg} \mathrm{s}^{-1} \mathrm{~cm}^{-2} \AA^{-1}$; line fluxes in units of $10^{-13} \mathrm{erg} \mathrm{s}^{-1} \mathrm{~cm}^{-2}$. The EUV flux is given in units of counts per second

\begin{tabular}{lcccc}
\hline feature & mean flux & rms flux & $R_{\max }$ & $F_{\text {var }}$ \\
\hline$U$ & 24.80 & 2.37 & 1.48 & 0.0865 \\
$B$ & 20.08 & 1.56 & 1.44 & 0.0697 \\
$V$ & 17.76 & 0.91 & 1.38 & 0.0421 \\
$R$ & 17.54 & 0.78 & 1.34 & 0.0406 \\
$I$ & 13.15 & 0.69 & 1.44 & 0.0557 \\
$F_{\lambda}(5100 \AA)$ & 14.4 & 0.9 & 1.36 & 0.040 \\
$\mathrm{H} \beta$ & 10.5 & 0.2 & 1.11 & -0.005 \\
$\mathrm{H} \alpha$ & 38.7 & 1.2 & 1.13 & -0.006 \\
$\mathrm{EUV}$ & 0.0993 & 0.0376 & 59 & 0.2035 \\
\hline
\end{tabular}

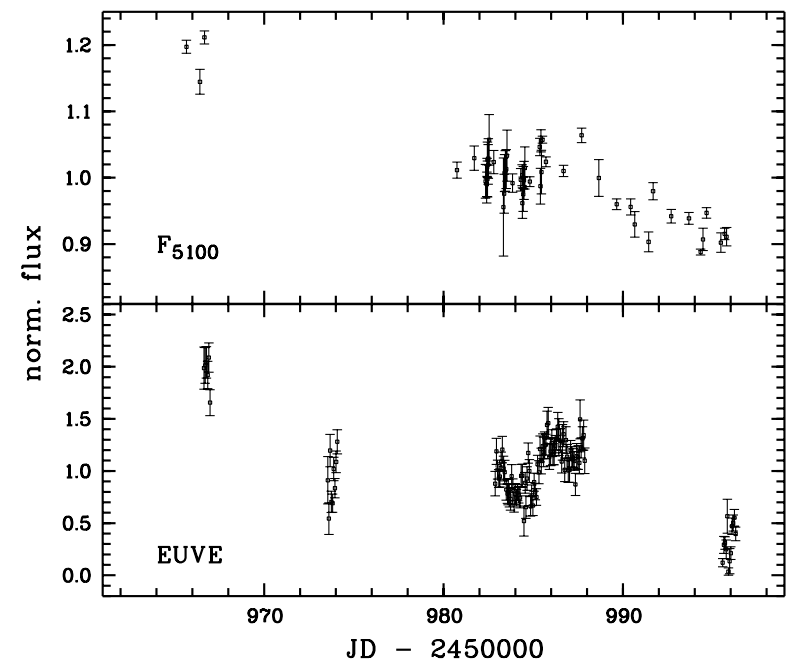

Fig. 4. The normalized EUVE light curve from the monitoring program for NGC 5548 (Chiang et al. 2000) is shown in the bottom panel. In the top panel the normalized variations of $F_{\lambda}(5100 \AA)$ are displayed

\subsection{Time-series analysis}

The emission lines are expected to change in response to variations in the far-UV continuum, primarily in response to variations at the unobservable wavelengths just shortward of $912 \AA$. Generally, it is necessary to assume that the observable continuum can approximate the behavior of the ionizing continuum and that the shortest observed UV wavelengths provide the best observable approximation to the "driving" (ionizing) continuum. However, for the first time we can make use of a well-sampled simultaneous X-ray light curve on short timescales (Chiang et al. 2000). The EUVE light curve and the variations of the optical continuum show very similar variability pattern (Figs. 3 and 4).

However, the $\mathrm{H} \alpha$ and $\mathrm{H} \beta$ emission line fluxes show only marginal indications for variations. Within the errors the emission line flux was constant within $5 \%$ which is also indicated by $F_{\text {var }}$ (cf. Table 7 ). In spite of this
Table 8. Sampling characteristics and full width at half maximum $(F W H M)$ of ACF and CCF features. The FWHM is given in units of days

\begin{tabular}{lccccc}
\hline feature & $N$ & \multicolumn{2}{c}{$F W H M(\mathrm{ACF})$} & \multicolumn{2}{c}{$F W H M(\mathrm{ICCF})$} \\
& & orig. & detr. & orig. & detr. \\
\hline$U$ & 120 & 6.8 & 3.3 & 7.1 & 5.0 \\
$B$ & 120 & 5.7 & 4.2 & 6.6 & 5.9 \\
$V$ & 178 & 1.7 & 0.2 & 9.3 & 2.2 \\
$R$ & 120 & 1.8 & 1.6 & 4.1 & 5.9 \\
$I$ & 120 & 0.2 & 0.2 & 3.6 & 3.7 \\
$F_{\lambda}(5100 \AA)$ & 52 & 30 & 0.4 & 6.1 & 3.3 \\
$\mathrm{H} \beta$ & 52 & 1.7 & 0.6 & 4.0 & 2.4 \\
$\mathrm{H} \alpha$ & 38 & 1.2 & 0.2 & 1.1 & 1.4 \\
\hline
\end{tabular}

we calculated cross-correlation functions. We used the extrem ultraviolet light curve measured with EUVE for the driving continuum kindly provided by Chiang and collaborators. We computed the ICCF (cf. White \& Peterson 1994) in the formalism of the so called "local CCF" (Welsh 1999). This approach takes into account the influence of the bias to underestimate the delay as has been shown by Welsh (1999). To correct for the influence of low frequency power in the flux variations we calculated a linear trend which was subtracted. The removal of a linear trend helps to reduce the bias of the observed delay towards values smaller than the real delay (Welsh 1999). We also applied the cross-correlation analysis to the detrended light curves, and we calculated the discrete correlation function (DCF) for comparison. Uncertainties in the ICCF results for the cross-correlation maxima and centroids were computed through Monte Carlo techniques (cf. Peterson et al. 1998).

The sampling characteristics of each of the light curves are given in Table 8 . The name of the feature and the total number of points, $N$, in the light curves are given that were used in computing the auto- and cross-correlation functions. The width $(F W H M)$ of the ACF based on the original and of the detrended light curves are given as well. The corresponding widths of ICCF computed by crosscorrelation with the EUV continuum at $\sim 0.2 \mathrm{keV}$ is listed too.

The results of the cross-correlation analysis are given in Table 9. The first column indicates the "responding" light curve (i.e., the light curve that is assumed to be responding to the driving light curve). The second and third column provide the peak value of the correlation coefficient $r_{\max }$ for the ICCF (original and detrended). The position of the peak of the cross-correlation functions $\tau_{\max }$ was measured by determing the location of the peak value of the ICCF and DCF; these values are given in the next four columns, respectively, The centroids $\tau_{\text {cent }}$ of the ICCF of the original and detrended light curves were computed using the points in the cross-correlation function with values greater than $0.6 r_{\max }$. The error estimate for the position of the cross-correlation peak $\Delta \tau_{\max }$ and 


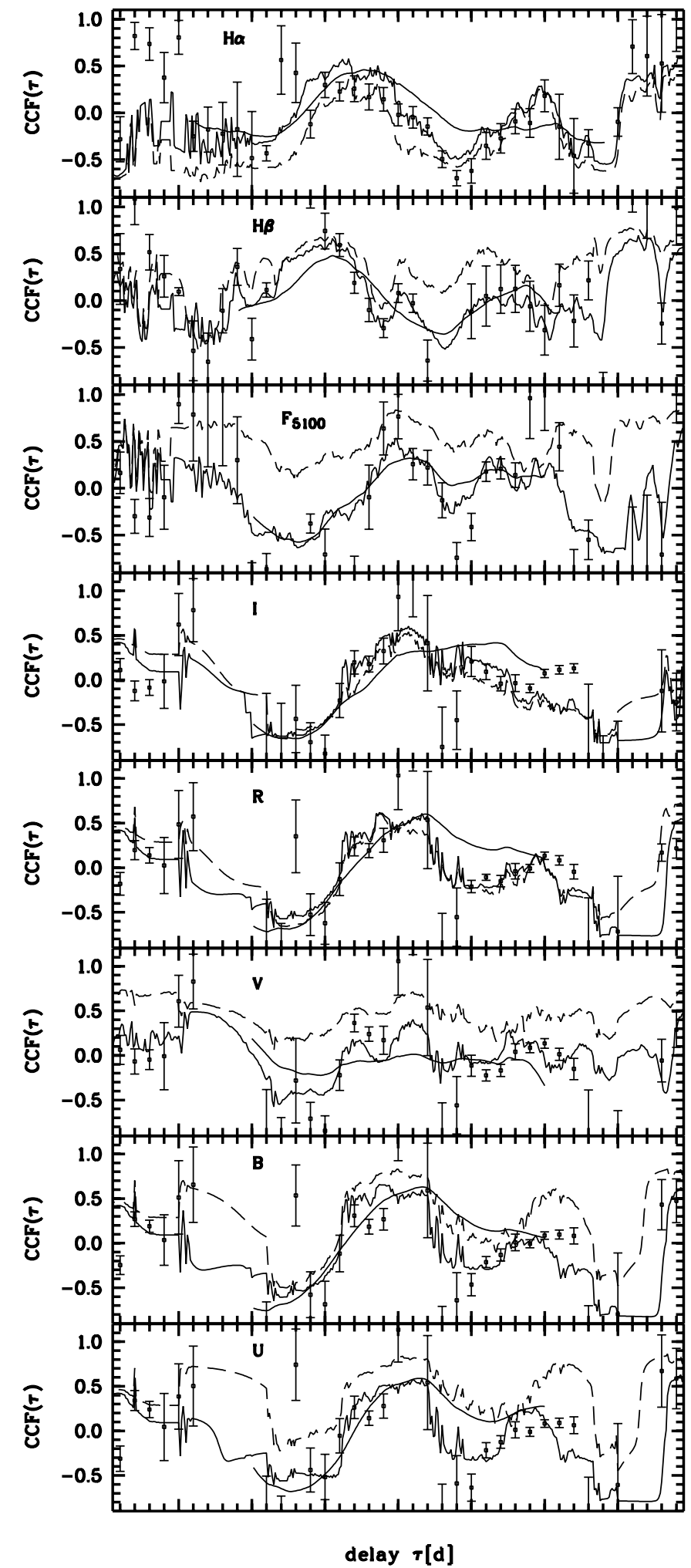

Fig. 5. Cross-correlation functions for $U, B, V, R, I$ fluxes, $F_{\lambda}(5100 \AA)$, and the $\mathrm{H} \beta$ and $\mathrm{H} \alpha$ emission line (from bottom to top). The ICCF computed with the original light curves (dashed line) and the ICCF based on the detrended light curves (solid line) are displayed. The average ICCFs which were obtained by estimating the uncertainties of the delays are shown as short solid line but restricted to $-10<\tau<10 \mathrm{~d}$. The discrete points show the DCF computed for each detrended light curve the ICCF centroid $\Delta \tau_{\text {cent }}$ for the original and detrended light curves are also given (cf. Table 9 columns $\Delta \tau_{\text {orig. }}$ and $\left.\Delta \tau_{\text {detr. }}\right)$.

The broad-band $(U, B, V, R, I)$ and the optical continuum flux variations appear to be simultaneous within the errors, relative to the EUV continuum variations at $\sim 0.2 \mathrm{keV}$ (Fig. 5). Taking into account the small variability amplitude expressed by $F_{\text {var }}$ (Table 7 ), the temporal coverage of the time series of 30 days only, with dense temporal sampling solely for the second half, the small CCF amplitudes, and the uncertainty of the location of the peak and centroid of the CCFs of $\approx 3$ days (Table 9 ) no reliable delay was detected for the broad emission lines of $\mathrm{H} \alpha$ and $\mathrm{H} \beta$ in response to the EUV variations (Fig. 5).

\subsection{Mean and root-mean-square spectra}

We calculated mean and root-mean-square (rms) spectra from the flux-scaled spectra for the $\mathrm{H} \alpha$ and $\mathrm{H} \beta$ regions. The rms spectrum is useful for isolating variable parts of line profiles.

The mean and rms spectra of the samples C, L1, L2, $\mathrm{W}$ are identical within less than $3 \%(\mathrm{H} \beta)$ and $1.5 \%(\mathrm{H} \alpha)$.

The mean and rms spectra of the $\mathrm{H} \alpha$ and $\mathrm{H} \beta$ region are presented for the spectra of subsample C (Fig. 6). It provides 19 epochs during the studied period and a daily sampling with homogeneous settings for the second half of June 1998. The rms spectrum of $\mathrm{H} \beta$ and $\mathrm{H} \alpha$ indicates continuum variations of the order of less than $5 \%$. Furthermore, weak broad emission line flux variations are superimposed but the amplitude is less than $2 \%$. Thus, these features in the rms-spectra again should be taken only as weak indications for emission line flux variations on timescales of days. However, the width of the broad HeII 4686 emission line in the rms spectrum is similar to that detected by Peterson et al. (2000) for the narrowline Seyfert 1 galaxy NGC 4051. The contribution of FeII emission (mulitplets 37, 38) should be negligible since there is no indication of variable FeII emission (multiplets 48,49$)$ in the rms spectrum in the wavelength range $\lambda \lambda 5200-5500 \AA$.

The spectra obtained at Calar Alto Observatory have a significant higher spectral resolution $\left(R \simeq 8 \mathrm{~km} \mathrm{~s}^{-1}\right)$ than the spectra of the other samples $\left(R \simeq 500 \mathrm{~km} \mathrm{~s}^{-1}\right)$. Hence, we used the spectra of sample $\mathrm{G}$ to search for small features as suggested by Stella (1990). The $\mathrm{H} \beta$ profile was filtered with a narrow gaussian curve $(F W H M=0.1 \AA)$ to improve the signal-to-noise ratio. The average spectra of each night of the 15 epochs obtained at Calar Alto Observatory are presented in Fig. 7 as well as the mean spectrum of the samples C, L1, L2, W. The shape of the $\mathrm{H} \beta$ profile is smooth and no narrow features have been detected during this short period. There is no obvious narrow moving feature visible in the $\mathrm{H} \beta$ profile. However, at $\lambda \simeq 4962 \AA$ there might be a marginal indication of a weak structure which is visible in the mean spectrum and in most of the individual spectra of sample G. It is 

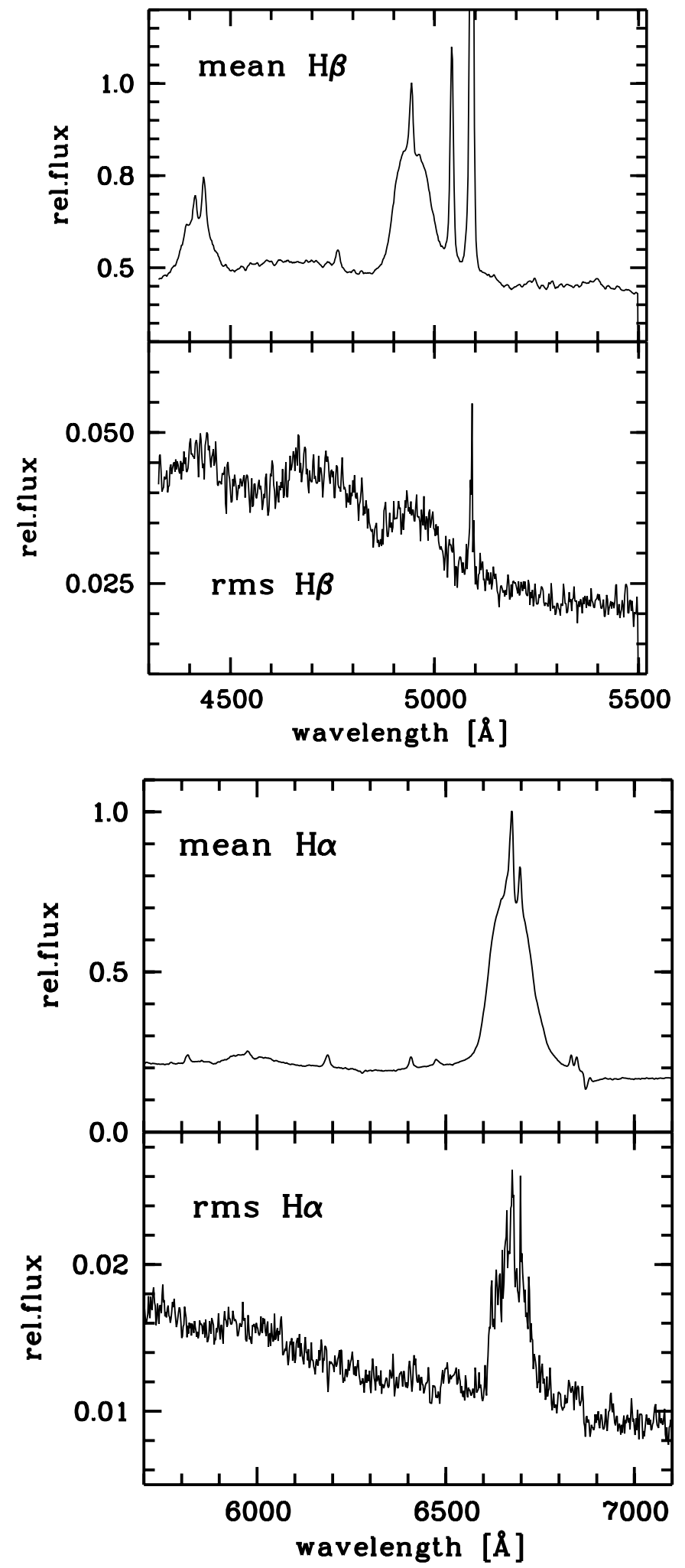

Fig. 6. The mean and root-mean-square (rms) spectra the of the $\mathrm{H} \beta$ (top) and $\mathrm{H} \alpha$ (bottom) line region are shown for sample C. The vertical scales are arbitrary. The rms spectrum of $\mathrm{H} \beta$ and $\mathrm{H} \alpha$ provide some indication for emission line variations but only of the order of less than $2 \%$. Note the broad feature at the location of HeII 4686 in the rms spectrum of the $\mathrm{H} \beta$ wavelength region. It is significantly broader than those of the $\mathrm{H} \beta$ or $\mathrm{H} \gamma$ line

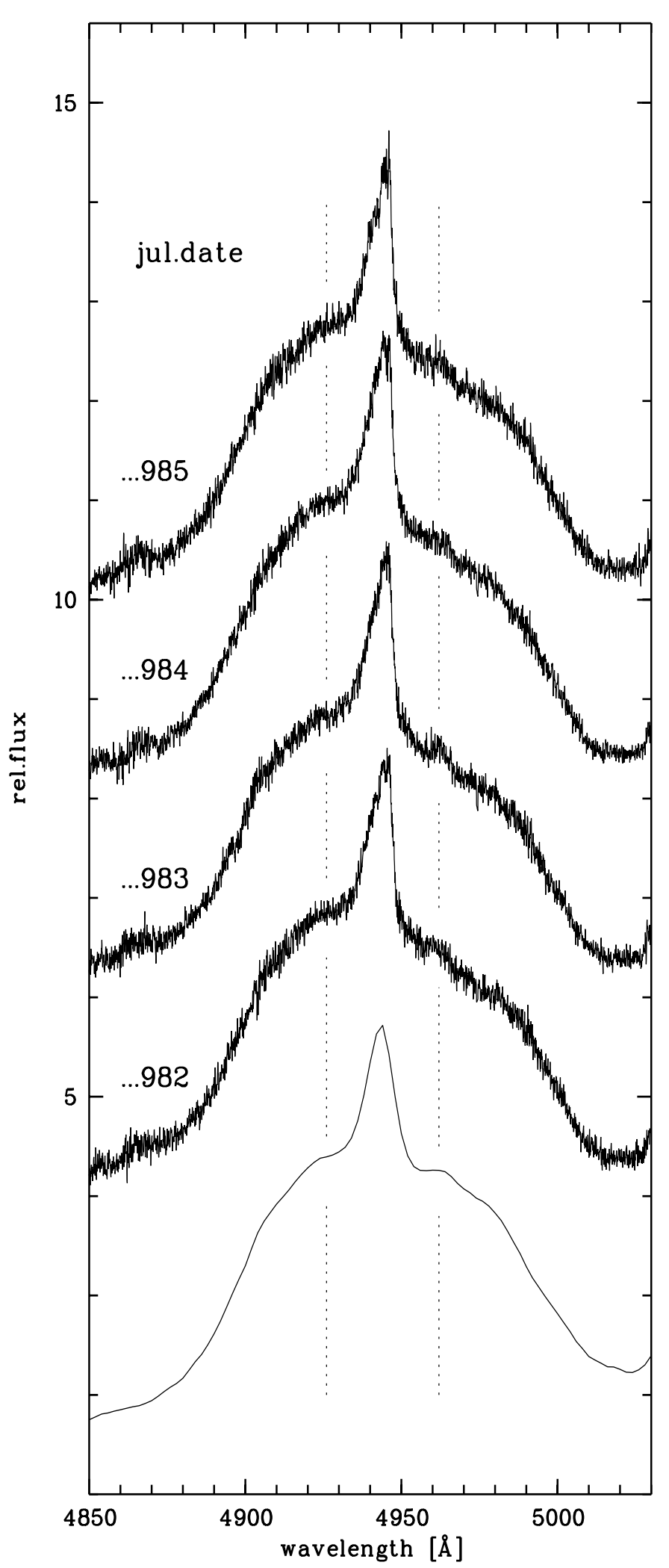

Fig. 7. The average echelle spectra of the $\mathrm{H} \beta$ emission line profile as obtained at Calar Alto Observatory are shown for each night. For comparison the mean spectrum obtained from the samples C, L, W with lower spectal resolution is displayed at the bottom of the panel. The vertical dotted lines indicate the location of the marginal feature at $\lambda \simeq 4962 \AA$ as well as the corresponding blueshifted location 
Table 9. Results of the cross-correlation analysis for the orginal and detrended light curves

\begin{tabular}{lcccccccccc}
\hline & \multicolumn{2}{c}{$r_{\max }$} & \multicolumn{4}{c}{$\tau_{\max }[$ days $]$} & \multicolumn{2}{c}{$\tau_{\text {cent }}[$ days $]$} & \multicolumn{2}{c}{ error.est. } \\
feature & orig. & detr. & \multicolumn{2}{c}{ ICCF } & \multicolumn{2}{c}{ DCF } & \multicolumn{2}{c}{ ICCF } & \multicolumn{2}{c}{ [days] } \\
& & & orig. & detr. & orig. & detr. & orig. & detr. & $\Delta \tau_{\text {orig. }}$ & $\Delta \tau_{\text {detr. }}$ \\
\hline$U$ & 0.86 & 0.57 & 0.0 & 0.9 & 0.0 & 1.0 & -0.1 & -0.1 & 0.6 & 1.1 \\
$B$ & 0.83 & 0.65 & -0.1 & -1.0 & 0.0 & 1.0 & -0.4 & -0.6 & 0.6 & 0.6 \\
$V$ & 0.71 & 0.40 & 1.0 & 1.0 & -2.0 & 1.0 & 1.4 & 1.0 & 2.1 & 2.1 \\
$R$ & 0.60 & 0.62 & -1.2 & -1.3 & 1.0 & 1.0 & -0.0 & -0.1 & 0.9 & 0.8 \\
$I$ & 0.52 & 0.58 & 0.5 & 0.5 & 1.0 & 1.0 & 0.3 & 0.6 & 5.2 & 2.0 \\
$F_{\lambda}(5100 \mathrm{~A})$ & 0.83 & 0.53 & -0.1 & -0.1 & 0.0 & 0.0 & 0.5 & 0.1 & 1.4 & 3.2 \\
$\mathrm{H} \beta$ & 0.56 & 0.04 & 6.1 & 6.5 & 6.0 & 7.0 & 6.1 & 7.8 & 3.0 & 4.5 \\
$\mathrm{H} \alpha$ & 0.26 & 0.29 & 9.8 & 9.7 & 10. & 10. & 9.8 & 9.7 & 6.0 & 2.7 \\
\hline
\end{tabular}

redshifted by $\sim 1100 \mathrm{~km} \mathrm{~s}^{-1}$ and the width amounts to $F W H M \simeq 6 \AA$ corresponding $\Delta v \simeq 350 \mathrm{~km} \mathrm{~s}^{-1}$. We used also the spectra of the samples with lower spectral resolution to search for this weak feature. It is also detectable in the mean spectrum based on these samples as can be seen in Fig. 7. Unfortunately, the redshift of $v \cong 1100 \mathrm{~km} \mathrm{~s}^{-1}$ places this weak feature in the red wing of the prominent [N II] 6583 emission line. Hence, it is not possible to detect this structure in the $\mathrm{H} \alpha$ line profile to provide further evidence for the existence of this weak feature.

\section{Summary}

The results of a short optical monitoring campaign (June 1998) on the Seyfert 1 galaxy NGC 5548 are presented in this paper. The principal findings are as follows:

1. The broad-band $(U, B, V)$ fluxes, and the optical continuum measured from spectrophotometry $F_{\lambda}(5100 \AA)$, showed a monotonic decrease of approximately $35 \%(U), 25 \%(B), 15 \%(V)$, and $30 \%$ $\left(F_{\lambda}(5100 \AA)\right)$ from the beginning to the end of the campaign ( $\sim 30$ days $)$;

2 . The broad-band $(R, I)$ fluxes, and the integrated emission-line fluxes of $\mathrm{H} \alpha$ and $\mathrm{H} \beta$ showed only marginal indications for variations on timescales of the order of a week with amplitudes less than $5 \%$;

3. In all broad band fluxes a short flare like event was detected during the night of June 22 (JD 2450987) which lasted $\approx 90 \mathrm{~min}$;

4. The shape of the variability pattern of the optical continuum is very similar to the detected variations in the EUV energy range $(\sim 0.2 \mathrm{keV})$ with EUVE by Chiang et al. (2000). But $F_{\text {var }}$ and $R_{\max }$ of the EUV variations are significantly larger than $F_{\text {var }}$ and $R_{\max }$ of $F_{\lambda}(5100 \AA)$;

5. The parameter $F_{\text {var }}$, which is essentially the rms variation about the mean, increased with decreasing wavelength for the broad-band measurements. The optical continuum $F_{\lambda}(5100 \AA)$ and the $V$ - and $R$-band exhibits comparable $F_{\text {var }}$.

The Balmer emission lines $\mathrm{H} \alpha$ and $\mathrm{H} \beta$ underwent no significant variations as measured by $F_{\text {var }}$ during this campaign within the errors;

6 . The variations of the broad-band and optical continuum fluxes are simultaneous with the $\sim 0.2 \mathrm{keV}$ variations. In spite of the Balmer lines $\mathrm{H} \alpha$ and $\mathrm{H} \beta$ showing no significant variability, we calculated crosscorrelation functions. No reliable delay was detected for $\mathrm{H} \alpha$ and $\mathrm{H} \beta$ with respect to the EUV continuum within the uncertainties;

7. The $\mathrm{H} \beta$ line profile was examined for narrow features. There is marginal evidence for a weak feature at $\lambda \simeq$ $4962 \AA$ with $F W H M \simeq 6 \AA$ which is redshifted by $\Delta v \simeq 1100 \mathrm{~km} \mathrm{~s}^{-1}$ with respect to $\mathrm{H} \beta_{\text {narrow }}$.

Acknowledgements. This work has been supported by SFB328D (Landessternwarte Heidelberg), by the NASA grant NAG5-3234 (University of Florida), the Russian Basic Research Foundation grant N94-02-4885a, N97-02-17625 (Sternberg Astronomical Institute, Special Astrophysical Observatory), by the Smithsonian Institution, and by INTAS grant N96-032. We would like to thank the FLWO remote observers P. Berlind and M. Calkins, and also S. Tokarz for help in reducing and archiving the FLWO data.

\section{References}

Allen, C. W. 1973, Astrophysical Quantities (The Athlone Press)

Alloin, D., Clavel, J., Peterson, B. M., Reichert, G. A., \& Stirpe, G. M. 1994, in Frontiers of Space and Ground-Based Astronomy, ed. W. Wamsteker, M. S. Longair, \& Y. Kondo (Dordrecht: Kluwer), 423

Alloin, D., Santos-Lleó, M., Peterson, B. M., et al. 1995, A\&A, 293, 293

Barr, P., \& Mushotzky, R. F. 1986, Nature, 320, 421

Carini, M. T., Noble, J. C., \& Miller, H. R. 1998, AJ, 116, 2667

Chiang, J., Reynolds, C. S., Blaes, O. M., et al. 2000, ApJ, 528,292

Clavel, J., Reichert, G. A., Alloin, D., et al. 1991, ApJ, 366, 64 Collier, S. J., Horne, K., Kaspi, S., et al. 1998, ApJ, 500, 162

Crenshaw, D. M., Rodríguez-Pascual, P. M., Penton, S. V., et al. 1996, ApJ, 470, 322 
Dietrich, M., Kollatschny, W., Peterson, B. M., et al. 1993, ApJ, 408, 416

Dietrich, M., Peterson, B. M., Albrecht, P., et al. 1998, ApJS, 115,185

Dultzin-Hacyan, D., Schuster, W. J., Parrao, L., et al. 1992, AJ, 103, 1769

Dultzin-Hacyan, D., Ruelas-Mayorga, A., \& Costero, R. 1993, Rev. Mex. Astron. Astrof., 25, 143

Dumont, A. M., \& Collin-Souffrin, S. 1990, A\&A, 229, 313

Edelson, R. A., Alexander, T., Crenshaw, D. M., et al. 1996, ApJ, 470, 364

Edelson, R. A., Koratkar, A., Nandra, K., et al. 2000, ApJ, 534,180

Eracleous, M., \& Halpern, J. P. 1993, ApJ, 409, 584

George, I. M., Mushotzky, R. F., Turner, T. J., et al. 1998a, ApJ, 509, 146

George, I. M., Turner, T. J., Netzer, H., et al. 1998b, ApJS, 114,73

Ghosh, K. K., Ramsey, B. D., Sadun, A. C., \& Soundararajaperumal, S. 2000, ApJS, 127, 11

Halpern, J. P. 1990, ApJ, 365, L51

Hubeny, I., Agol, E., Blaes, O., \& Krolik, J. H. 2000, ApJ, 533, 710

Jang, M., \& Miller, H. R. 1997, AJ, 114, 565

Kaspi, S., Maoz, D., Netzer, H., et al. 1996, ApJ, 470, 336

Kaspi, S., Smith, P. S., Netzer, H., et al. 2000, ApJ, 533, 631

Korista, K. T., Alloin, D., Barr, P., et al. 1995, ApJS, 97, 285

Laor, A., \& Netzer, H. 1989, MNRAS, 238, 897

Leighly, K. M., Kunieda, H., Awaki, H. et al. 1996, ApJ, 463, 158

Leighly, K. M., O’Brien, P. T., Edelson, R. A., et al. 1997, ApJ, 483, 767

Lyuty, V. M. 1972, Astron. Zh, 49, 930

Lyuty, V. M., Aslanov, A. A., Khruzina, T. S., et al. 1989, Sov. Astron. Lett., 15, 247

Maoz, D., Netzer, H., Peterson, B. M., et al. 1993, ApJ, 404, 576

Marshall, H. L., Carone, T. E., Peterson, B. M., et al. 1997, ApJ, 479, 222

Merkulova, N. I. 2000, AJ, 119, 631

Nandra, K., Clavel., J., Edelson, R. A., et al. 1998, ApJ, 505, 594

Neckel, T., \& Chini, R. 1980, A\&AS, 39, 411

Netzer, H., \& Peterson, B. M. 1997, in Astronomical Time Series, ed. D. Maoz, A. Sternberg, \& E. Leibowitz (Dordrecht: Kluwer), 85

Noble, J. C., Carini, M. T., Miller, H. R., \& Goodrich, B. 1997, AJ, 113, 1995
O'Brien, P. T., Dietrich, M., Leighly, K. M., et al. 1998, ApJ, 509, 163

Penston, M. J., Penston, M. V., \& Sandage, A. 1971, PASP, 83, 783

Peterson, B. M., \& Collins, G. W., II. 1983, ApJ, 270, 71

Peterson, B. M., Balonek, T. J., Barker, E. S., et al. 1991, ApJ, 368, 119

Peterson, B. M., Alloin, D., Axon, D., et al. 1992, ApJ, 392, 470

Peterson, B. M. 1993, PASP, 105, 247

Peterson, B. M., Berlind, P., Bertram, R., et al. 1994, ApJ, 425,622

Peterson, B. M., Pogge, R. W., Wanders, I., Smith, S. M., \& Romanishin, W. 1995, PASP, 107, 579

Peterson, B. M., Wanders, I., Horne, K., et al. 1998, PASP, 110,660

Peterson, B. M., Barth, A. J., Berlind, P., et al. 1999, ApJ, 510,659

Peterson, B. M., McHardy, I. M., Wilkes, B. J., et al. 2000 , ApJ, 542, 161

Petrucci, P. O., Chelli, A., Henri, G., et al. 1999, A\&A, 342, 687

Piirola, V. 1973, A\&A, 27, 383

Reichert, G. A., Rodríguez-Pascual, P. M., Alloin, D., et al. 1994, ApJ, 425, 582

Rodríguez-Pascual, P. M., Alloin, D., Clavel, J., et al. 1997, ApJS, 110, 9

Romanishin, W., Balonek, T. J., Ciardullo, R., et al. 1995, ApJ, 455, 516

Santos-Lleó, M., Chatzichristou, E., Mendes de Oliveira, C., et al. 1997, ApJS, 112, 271

Stella, L. 1990, Nature, 344, 747

Stirpe, G. M., Winge, C., Altieri, B., et al. 1994, ApJ, 425, 609

van Groningen, E., \& Wanders, I. 1992, PASP, 104, 700

Wandel, A., Peterson, B. M., \& Malkan, M. A. 1999, ApJ, 526, 579

Wanders, I., Peterson, B. M., Alloin, D., et al. 1997, ApJS, 113,69

Wamsteker, W. 1981, A\&A, 97, 329

Warwick, R., Smith, D. A., Yaqoob, T., et al. 1996, ApJ, 470, 349

Welsh, W. F., Peterson, B. M., Koratkar, A. P., \& Korista, K. T. 1998, ApJ, 509, 118

Welsh, W. F. 1999, PASP, 111, 1347

White, R. J., \& Peterson, B. M. 1994, PASP, 106, 879

Xanthopoulos, E., \& DeRobertis, M. M. 1991, PASP, 103, 378

Zheng, W., Veilleux, S., \& Grandi, S. A. 1991, ApJ, 381, 121 
Table 2A. log of the spectroscopic observations

\begin{tabular}{|c|c|c|c|c|c|c|c|c|c|}
\hline civil date & $\begin{array}{c}\text { jul.date } \\
\text { mid. }\end{array}$ & code & $\begin{array}{r}t_{\text {int }} \\
{[\mathrm{s}]}\end{array}$ & $\begin{array}{c}\text { range } \\
{[\AA]}\end{array}$ & $\begin{array}{c}\text { aperture } \\
\text { [arcsec] }\end{array}$ & $\begin{array}{c}\mathrm{PA} \\
\circ\end{array}$ & $\begin{array}{c}\text { see. } \\
{[\operatorname{arcsec}]}\end{array}$ & $\begin{array}{l}\text { Res. } \\
{[\AA]}\end{array}$ & filename \\
\hline 98 Jun. 01 & 2450965.67125 & $\mathrm{C}$ & 180 & $3655-7529$ & $3.0 \times 4.6$ & 91 & 2.0 & & n50965f \\
\hline 98 Jun. 01 & 2450966.42281 & $\mathrm{~W}$ & 3600 & $4312-5612$ & $3.0 \times 11.0$ & 90 & 2.0 & $7-8$ & n50966wb \\
\hline 98 Jun. 02 & 2450966.67348 & $\mathrm{C}$ & 180 & $3654-7529$ & $3.0 \times 4.6$ & 91 & 3.0 & & n50966f \\
\hline 98 Jun. 16 & 2450980.75052 & $\mathrm{C}$ & 180 & $3655-7530$ & $3.0 \times 4.6$ & 90 & 2.0 & & n50980f \\
\hline 98 Jun. 17 & 2450981.71094 & $\mathrm{C}$ & 180 & $3655-7530$ & $3.0 \times 4.6$ & 90 & 1.5 & & n50981f \\
\hline 98 Jun. 17 & 2450982.33950 & $\mathrm{~W}$ & 3720 & $4298-5598$ & $3.0 \times 11.0$ & 90 & 3.5 & $7-8$ & n50982wba \\
\hline 98 Jun. 17 & 2450982.40486 & G & 3600 & $5600-7100$ & $2.05 \times 2.61$ & 0 & 2 & 0.12 & n50982da1 \\
\hline 98 Jun. 17 & 2450982.40486 & G & 3600 & $4375-5375$ & $2.05 \times 2.61$ & 0 & 2 & 0.12 & n50982db1 \\
\hline 98 Jun. 17 & 2450982.41389 & L1 & 1200 & $4090-5800$ & $8.0 \times 6.6$ & 0 & 2 & 9 & n50982llb1 \\
\hline 98 Jun. 17 & 2450982.41389 & L1 & 1200 & $4090-5800$ & $8.0 \times 19.8$ & 0 & 2 & 9 & n50982llb4 \\
\hline 98 Jun. 17 & 2450982.42847 & L1 & 1200 & $4090-5800$ & $8.0 \times 6.6$ & 0 & 2 & 9 & n509821lb2 \\
\hline 98 Jun. 17 & 2450982.42847 & L1 & 1200 & $4090-5800$ & $8.0 \times 19.8$ & 0 & 2 & 9 & n50982llb5 \\
\hline 98 Jun. 17 & 2450982.44514 & L1 & 1200 & $4090-5800$ & $8.0 \times 6.6$ & 0 & 2 & 9 & n50982llb3 \\
\hline 98 Jun. 17 & 2450982.44514 & L1 & 1200 & $4090-5800$ & $8.0 \times 19.8$ & 0 & 2 & 9 & n50982llb6 \\
\hline 98 Jun. 17 & 2450982.45000 & G & 3600 & $5600-7100$ & $2.05 \times 2.61$ & 0 & 2 & 0.12 & n50982da2 \\
\hline 98 Jun. 17 & 2450982.45000 & G & 3600 & $4375-5375$ & $2.05 \times 2.61$ & 0 & 2 & 0.12 & n50982db2 \\
\hline 98 Jun. 17 & 2450982.48648 & $\mathrm{~W}$ & 5505 & $4296-5586$ & $3.0 \times 11.0$ & 90 & 3.4 & $7-8$ & n50982wbb \\
\hline 98 Jun. 17 & 2450982.49514 & G & 3600 & $5600-7100$ & $2.05 \times 2.61$ & 0 & 2 & 0.12 & n50982da3 \\
\hline 98 Jun. 17 & 2450982.49514 & G & 3600 & $4375-5375$ & $2.05 \times 2.61$ & 0 & 2 & 0.12 & n50982db3 \\
\hline 98 Jun. 18 & 2450982.53958 & $\mathrm{G}$ & 3600 & $5600-7100$ & $2.05 \times 2.61$ & 0 & 2 & 0.12 & n50982da4 \\
\hline 98 Jun. 18 & 2450982.53958 & G & 3600 & $4375-5375$ & $2.05 \times 2.61$ & 0 & 2 & 0.12 & n50982db4 \\
\hline 98 Jun. 18 & 2450982.79700 & $\mathrm{C}$ & 180 & $3656-7530$ & $3.0 \times 4.6$ & 90 & 1.5 & & n50982f \\
\hline 98 Jun. 18 & 2450983.33958 & L1 & 1200 & $4090-5800$ & $8.0 \times 6.6$ & 0 & 2 & 9 & n50983llb1 \\
\hline 98 Jun. 18 & 2450983.33958 & L1 & 1200 & $4090-5800$ & $8.0 \times 19.8$ & 0 & 2 & 9 & n509831lb2 \\
\hline 98 Jun. 18 & 2450983.37845 & $\mathrm{~W}$ & 4235 & $4284-5586$ & $3.0 \times 11.0$ & 90 & 3.0 & $7-8$ & n50983wb \\
\hline 98 Jun. 18 & 2450983.39861 & G & 3600 & $5600-7100$ & $2.05 \times 2.61$ & 0 & 2 & 0.12 & n50983da1 \\
\hline 98 Jun. 18 & 2450983.39861 & G & 3600 & $4375-5375$ & $2.05 \times 2.61$ & 0 & 2 & 0.12 & n50983db1 \\
\hline 98 Jun. 18 & 2450983.44375 & G & 3600 & $5600-7100$ & $2.05 \times 2.61$ & 0 & 2 & 0.12 & n50983da2 \\
\hline 98 Jun. 18 & 2450983.44375 & G & 3600 & $4375-5375$ & $2.05 \times 2.61$ & 0 & 2 & 0.12 & n50983db2 \\
\hline 98 Jun. 18 & 2450983.49236 & G & 3600 & $5600-7100$ & $2.05 \times 2.61$ & 0 & 2 & 0.12 & n50983da3 \\
\hline 98 Jun. 18 & 2450983.49236 & G & 3600 & $4375-5375$ & $2.05 \times 2.61$ & 0 & 2 & 0.12 & n50983db3 \\
\hline 98 Jun. 19 & 2450983.53681 & G & 3600 & $5600-7100$ & $2.05 \times 2.61$ & 0 & 2 & 0.12 & n50983da4 \\
\hline 98 Jun. 19 & 2450983.53681 & G & 3600 & $4375-5375$ & $2.05 \times 2.61$ & 0 & 2 & 0.12 & n50983db4 \\
\hline 98 Jun. 19 & 2450983.84502 & $\mathrm{C}$ & 180 & $3655-7530$ & $3.0 \times 4.6$ & 90 & 1.5 & & n50983f \\
\hline 98 Jun. 19 & 2450984.30069 & L1 & 2400 & $4090-5800$ & $8.0 \times 6.6$ & 0 & 4 & 9 & n50984llb1 \\
\hline 98 Jun. 19 & 2450984.30069 & L1 & 2400 & $4090-5800$ & $8.0 \times 19.8$ & 0 & 4 & 9 & n50984llb4 \\
\hline 98 Jun. 19 & 2450984.34167 & L1 & 2400 & $5630-7250$ & $8.0 \times 6.6$ & 0 & 4 & 9 & n50984llr1 \\
\hline 98 Jun. 19 & 2450984.34167 & L1 & 2400 & $5630-7250$ & $8.0 \times 19.8$ & 0 & 4 & 9 & n50984llr3 \\
\hline 98 Jun. 19 & 2450984.37847 & L1 & 2400 & $4090-5800$ & $8.0 \times 6.6$ & 0 & 4 & 9 & n50984llb2 \\
\hline 98 Jun. 19 & 2450984.37847 & L1 & 2400 & $4090-5800$ & $8.0 \times 19.8$ & 0 & 4 & 9 & n50984llb5 \\
\hline 98 Jun. 19 & 2450984.39514 & G & 3600 & $5600-7100$ & $2.05 \times 2.61$ & 0 & 1 & 0.12 & n50984da1 \\
\hline 98 Jun. 19 & 2450984.39514 & G & 3600 & $4375-5375$ & $2.05 \times 2.61$ & 0 & 1 & 0.12 & n50984db1 \\
\hline 98 Jun. 19 & 2450984.41389 & L1 & 2400 & $5630-7250$ & $8.0 \times 6.6$ & 0 & 4 & 9 & n50984llr2 \\
\hline 98 Jun. 19 & 2450984.41389 & L1 & 2400 & $5630-7250$ & $8.0 \times 19.8$ & 0 & 4 & 9 & n50984llr4 \\
\hline 98 Jun. 19 & 2450984.43958 & G & 3600 & $5600-7100$ & $2.05 \times 2.61$ & 0 & 1 & 0.12 & n50984da2 \\
\hline 98 Jun. 19 & 2450984.43958 & $\mathrm{G}$ & 3600 & $4375-5375$ & $2.05 \times 2.61$ & 0 & 1 & 0.12 & n50984db2 \\
\hline
\end{tabular}


Table 2A. continued

\begin{tabular}{|c|c|c|c|c|c|c|c|c|c|}
\hline civil date & $\begin{array}{c}\text { jul.date } \\
\text { mid. }\end{array}$ & code & $\begin{array}{c}t_{\text {int }} \\
{[\mathrm{s}]}\end{array}$ & $\begin{array}{c}\text { range } \\
{[\AA]}\end{array}$ & $\begin{array}{c}\text { aperture } \\
\text { [arcsec] }\end{array}$ & $\begin{array}{c}\mathrm{PA} \\
\circ\end{array}$ & $\begin{array}{c}\text { see. } \\
{[\operatorname{arcsec}]}\end{array}$ & $\begin{array}{l}\text { Res. } \\
{[\AA]}\end{array}$ & filename \\
\hline 98 Jun. 19 & 2450984.44403 & L1 & 1200 & $4090-5800$ & $8.0 \times 6.6$ & 0 & 4 & 9 & n50984llb3 \\
\hline 98 Jun. 19 & 2450984.44403 & L1 & 1200 & $4090-5800$ & $8.0 \times 19.8$ & 0 & 4 & 9 & n50984llb6 \\
\hline 98 Jun. 19 & 2450984.48403 & G & 3600 & $5600-7100$ & $2.05 \times 2.61$ & 0 & 1 & 0.12 & n50984da3 \\
\hline 98 Jun. 19 & 2450984.48403 & G & 3600 & $4375-5375$ & $2.05 \times 2.61$ & 0 & 1 & 0.12 & n50984db3 \\
\hline 98 Jun. 20 & 2450984.52778 & G & 3600 & $5600-7100$ & $2.05 \times 2.61$ & 0 & 1 & 0.12 & n50984da4 \\
\hline 98 Jun. 20 & 2450984.52778 & $\mathrm{G}$ & 3600 & $4375-5375$ & $2.05 \times 2.61$ & 0 & 1 & 0.12 & n50984db4 \\
\hline 98 Jun. 20 & 2450984.80627 & $\mathrm{C}$ & 180 & $3655-7530$ & $3.0 \times 4.6$ & 90 & 1.5 & & n50984f \\
\hline 98 Jun. 20 & 2450985.29444 & L1 & 2400 & $5630-7250$ & $8.0 \times 6.6$ & 0 & 2 & 9 & n50985llr1 \\
\hline 98 Jun. 20 & 2450985.29444 & L1 & 2400 & $5630-7250$ & $8.0 \times 19.8$ & 0 & 2 & 9 & n50985llr3 \\
\hline 98 Jun. 20 & 2450985.36319 & L1 & 2400 & $4090-5800$ & $8.0 \times 6.6$ & 0 & 2 & 9 & n50985llb1 \\
\hline 98 Jun. 20 & 2450985.36319 & L1 & 2400 & $4090-5800$ & $8.0 \times 19.8$ & 0 & 2 & 9 & n50985llb3 \\
\hline 98 Jun. 20 & 2450985.39653 & L1 & 2400 & $5630-7250$ & $8.0 \times 6.6$ & 0 & 2 & 9 & n50985llr2 \\
\hline 98 Jun. 20 & 2450985.39653 & L1 & 2400 & $5630-7250$ & $8.0 \times 19.8$ & 0 & 2 & 9 & n50985llr4 \\
\hline 98 Jun. 20 & 2450985.40174 & $\mathrm{G}$ & 4500 & $5600-7100$ & $2.05 \times 2.61$ & 0 & 1 & 0.12 & n50985da1 \\
\hline 98 Jun. 20 & 2450985.40174 & $\mathrm{G}$ & 4500 & $4375-5375$ & $2.05 \times 2.61$ & 0 & 1 & 0.12 & n50985db1 \\
\hline 98 Jun. 20 & 2450985.43056 & L1 & 2400 & $4090-5800$ & $8.0 \times 6.6$ & 0 & 2 & 9 & n50985llb2 \\
\hline 98 Jun. 20 & 2450985.43056 & L1 & 2400 & $4090-5800$ & $8.0 \times 19.8$ & 0 & 2 & 9 & n50985llb4 \\
\hline 98 Jun. 20 & 2450985.45660 & G & 4500 & $5600-7100$ & $2.05 \times 2.61$ & 0 & 1 & 0.12 & n50985da2 \\
\hline 98 Jun. 20 & 2450985.45660 & G & 4500 & $4375-5375$ & $2.05 \times 2.61$ & 0 & 1 & 0.12 & n50985db2 \\
\hline 98 Jun. 20 & 2450985.51215 & G & 4500 & $5600-7100$ & $2.05 \times 2.61$ & 0 & 1 & 0.12 & n50985da3 \\
\hline 98 Jun. 20 & 2450985.51215 & G & 4500 & $4375-5375$ & $2.05 \times 2.61$ & 0 & 1 & 0.12 & n50985db3 \\
\hline 98 Jun. 21 & 2450985.70999 & $\mathrm{C}$ & 180 & $3655-7530$ & $3.0 \times 4.6$ & 90 & 1.5 & & n50985f \\
\hline 98 Jun. 22 & 2450986.68704 & $\mathrm{C}$ & 180 & $3654-7529$ & $3.0 \times 4.6$ & 90 & 2.0 & & n50986f \\
\hline 98 Jun. 23 & 2450987.69251 & $\mathrm{C}$ & 180 & $3654-7529$ & $3.0 \times 4.6$ & 90 & 1.5 & & n50987f \\
\hline 98 Jun. 24 & 2450988.65380 & $\mathrm{C}$ & 210 & $3654-7529$ & $3.0 \times 4.6$ & 90 & 1.5 & & n50988f \\
\hline 98 Jun. 25 & 2450989.64822 & $\mathrm{C}$ & 180 & $3655-7529$ & $3.0 \times 4.6$ & 90 & 1.5 & & n50989f \\
\hline 98 Jun. 25 & 2450990.42708 & L2 & 1800 & $4090-5800$ & $2.0 \times 6.0$ & 0 & 2 & 8 & n50990llb \\
\hline 98 Jun. 26 & 2450990.65554 & $\mathrm{C}$ & 180 & $3658-7532$ & $3.0 \times 4.6$ & 90 & 1.5 & & n50990f \\
\hline 98 Jun. 26 & 2450991.42708 & L2 & 1800 & $4090-5800$ & $2.0 \times 6.0$ & 0 & 2 & 8 & n50991llb \\
\hline 98 Jun. 27 & 2450991.67628 & $\mathrm{C}$ & 180 & $3657-7531$ & $3.0 \times 4.6$ & 90 & 1.5 & & n50991f \\
\hline 98 Jun. 28 & 2450992.68902 & $\mathrm{C}$ & 180 & $3657-7531$ & $3.0 \times 4.6$ & 90 & 1.5 & & n50992f \\
\hline 98 Jun. 29 & 2450993.67738 & $\mathrm{C}$ & 180 & $3656-7531$ & $3.0 \times 4.6$ & 90 & 1.5 & & n50993f \\
\hline 98 Jun. 29 & 2450994.32743 & W & 3600 & $4294-5594$ & $3.0 \times 11.0$ & 90 & 2.2 & $7-8$ & n50994wba \\
\hline 98 Jun. 29 & 2450994.46063 & W & 3660 & $4298-5586$ & $3.0 \times 11.0$ & 90 & 2.8 & $7-8$ & n50994wbb \\
\hline 98 Jun. 30 & 2450994.65447 & $\mathrm{C}$ & 180 & $3656-7531$ & $3.0 \times 4.6$ & 90 & 1.5 & & n50994f \\
\hline 98 Jun. 30 & 2450995.45581 & W & 5500 & $4298-5588$ & $3.0 \times 11.0$ & 90 & 1.7 & $7-8$ & n50995wb \\
\hline 98 Jul. 01 & 2450995.66466 & $\mathrm{C}$ & 180 & $3659-7534$ & $3.0 \times 4.6$ & 90 & 1.5 & & n50995fa \\
\hline 98 Jul. 01 & 2450995.77620 & $\mathrm{C}$ & 180 & 3659-7534 & $3.0 \times 4.6$ & 90 & 1.5 & & n $50995 \mathrm{fb}$ \\
\hline
\end{tabular}

